\title{
M étodo como arte
}

\author{
Alberto M arradi
}

Catedrático de M etodología. U niversidad de Florencia (Italia)

\section{Sumario}

1. El término en el lenguaje ordinario M étodo y técnica

2. Conceptos de método en las reflexiones sobre la ciencia

3. M étodo, métodos, metodología
M etodología (y método)

entre gnoseología y técnica

Bibliografía

Estas breves reflexiones surgieron de la constatación, no nueva por cierto, de que al término método se le atribuyen - como a muchos otros términos- significados muy divergentes, y de la sensación de que ya es tiempo de revisar esta dispersión semántica, y tal vez también de manifestar y justificar alguna pre ferencia.

\section{El término en el lenguaje ordinario}

Iniciaré esta exploración de los significados del término desde el lenguaje ordinario. M e parece, en efecto, que la tesis - de Bachelard $(1934 ; 1938)$ y de muchos otros- acerca de la existencia de una separación neta (la coupure épis temologique) entre lenguaje ordinario y lenguaje científico es una de aquellas tesis autogratificantes que los científicos suscriben sin jamás preocuparse de someterla a control empírico.

En este párrafo quisiera traer un poco de agua al molino de la tesis contraria, aquélla de la plena continuidad entre lenguaje ordinario y lenguaje científico: dije sólo «un poco», porque las escasas citas que voy a presentar no pueden demostrar casi nada - ni aún acerca del término método.

Comenzaré entonces con una breve reseña - obviamente sin pretensiones de exhaustividad- de las acepciones del término que considero propias del lenguaje ordinario y que encontré en obras clásicas de la sociología. Q uien tenga a mano un diccionario puede constatar que en esta reseña de trazos clásicos, aunque breve, se encuentra prácticamente toda la gama de acepciones que el término asume en el lenguaje ordinario. 
a) Criterio: «C ada sociedad debe tener un método para distribuir entre sus miembros los bienes materiales que produce» (H omans, 1950: 275).

b) Forma: «Un noble tiene varios métodos de perder su status: la derrota militar, la venta de su título...» (Sorokin, 1947: 259); «N ada sería considerado más sospechoso que este método para expandir la producción» (G albraith, 1958: 106).

c) Camino (para conseguir un fin): «La fe en una dosis mayor de planificación y nacionalización como método de mejoramiento económico» (Goldthorpe y otros, 1968: 23); «El ideal de igualdad es fuerte, pero no tiene métodos regulados y garantizados para afirmarse» (C ooley, 1909: 14).

d) U so corriente, praxis: «Según el viejo método de pasar de madre a hija las nociones tradicionales de economía doméstica» (Lynd y Lynd, 1929: 157). «Los viejos métodos para inspirar respeto fueron cada vez menos usados» (Collins, 1975: 224); «Se está dejando de lado la erosión social atribuible al método actual de introducir rápidos cambios tecnológicos» (M erton, 1949: 325);

e) Procedimiento: «Todos los métodos conocidos de evaluar y calcular el tiempo comportan repeticiones» (Giddens, 1979: 204).

f) T écnica: «Los métodos para saciar el hambre y la sed se aprenden con la experiencia y la enseñanza» (H obhouse, 1906: 2) 2); «A causa de la mejora en los métodos agrícolas la tierra está en condiciones de alimentar una población mayor (Riesman y otros, 1950: 14).

g) Artificio, engaño: «Todos los métodos conocidos para deshacer una muchedumbre implican algún método para distraer la atención» (Park y Burgess, 1921: 876-77).

El prefijo met- $(\mu \varepsilon \tau \alpha)$ denuncia el origen griego de la palabra. Ese prefijo aparece en muchos términos académicos de las lenguas occidentales en sus diferentes significados: como «más allá» en metafísica, metástasis, metalenguaje; como «en lugar de» en metáfora, metatesis, metonimia, metamorfosis, metempsicosis. En combinación con el sustantivo odos ('camino'), el prefijo met- asu-

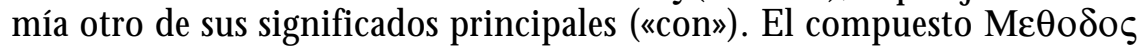
significaba, por lo tanto, 'camino con [el cual]'.

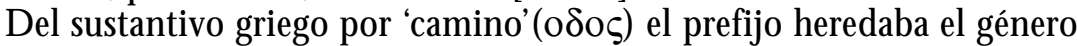
femenino, que luego transmitió a sus derivados francés y alemán.

El significado en el lenguaje ordinario griego clásico ('sucesión de actos tendentes a conseguir un fin') quedó fiel a la etimología del término.

A la luz de los ejemplos citados en los diccionarios griegos, no parece que el significado filosófico se separase del término ordinario, por ejemplo: es ordinaria la acepción según la cual Platón habla, en el Fedro, del método de H ipócrates y del método de los retóricos. Y la idea de recorrido, de la sucesión de pasos asociada al término, está presente en la difusión, en el griego postclásico, de la expresión - de algún modo redundante- $0 \tau \rho \circ \pi \varsigma \varsigma \tau \varepsilon \varsigma$ $\mu \varepsilon \tau o \delta o v$ ('la dirección del camino').

D espués de casi veinte siglos, en la definición que da del término la L ogique 
de Port Royal («ars bene disponendi seriem plurimarum cogitationum»: Arnauld e N icole, 1662), la idea de una sucesión de pasos sobrevive y se conjuga con la del arte (entendida en el sentido de habilidad).

\section{Conceptos de método en las reflexiones sobre la ciencia}

La acentuación de los aspectos intelectuales en el significado del término se evidencia ya cuando Jean Bodin dedica (1566) una reflexión específica al método de las ciencias histórico-políticas.

Primero en la tradición filosófica occidental, D escartes proclama el papel central del método en la actividad intelectual (D iscours de la M éthode, 1637). Ya él había definido el método como «reglas ciertas y fáciles que cualquiera que fuera el que las observara con exactitud le sería imposible tomar lo falso por verdadero, sin requerir inútilmente esfuerzos de la mente, pero aumentando siempre gradualmente el [propio] saber y lo conduciría al conocimiento verdadero de todo aquello que fuese capaz de conocer» (Cuarta Regula ad directionem ingenii, 1628).

Esta naturaleza universal del método había sido subrayada algunos años antes por Francis Bacon en el aforismo 122 del N ovum O rganum (1620): «N uestro método de investigación pone casi a la par todos los intelectos, porque deja poco espacio a las capacidades individuales, pero las liga con reglas muy solidas y con demostraciones».

Relacionando pacientemente las declaraciones de dos pensadores que son considerados como la raíz de dos direcciones contrapuestas en el pensamiento filosófico-científico contemporáneo (racionalismo y empirismo), impacta la extraordinaria similitud de sus conceptos de método:

- No estamos en presencia de algo esotérico y especializado. Es más, las reglas a seguir son fáciles, $\alpha \nu \tau o \mu\langle\tau \imath \chi \alpha \sigma$ («sin requerir effuerzos de la mente») al alcance de todos («de cualquiera», «pone a la par todos los intelectos»).

- No son requeridos ni rechazados los conocimientos especializados, las capacidades, las iniciativas personales ( «deja poco espacio a las capacidades individuales, pero las liga a reglas muy sólidas y con demostraciones»); las reglas se imponen para todos («reglas ciertas... observadas con exactitud»).

Si se siguen fielmente - agrega D escartes - las reglas de este método, «conducirán al conocimiento verdadero».1

En los dos o tres siglos siguientes, por efecto de los éxitos que la matematización de la ciencia va consiguiendo con Kepler, Galileo, N ewton, Boyle y Lavoisier, esta idea de «un método matemático universal, aplicable en todos los campos del conocimiento científico» asume connotaciones «pitagórico-pla

1. Carlos Strasser ha resaltado (1979: 19) otros rasgos que Bacon y D escartes tienen en común: el desprecio por todos los conocimientos que la sabiduría humana habría acumulado antes de ellos y el énfasis sobre la utilidad práctica de los descubrimientos. 
tónicas» (ver Berka, 1963: 8) que no tenía en Bacon y tenía sólo potencialmente en D escartes. Se hace cada vez más explícita la idea - implícita en el concepto griego de $\varepsilon \pi \imath \sigma \tau \eta \mu \eta$ - que verum y certum son conjugables, que se puede, por lo tanto, hablar de la realidad que nos circunda sin renunciar a la certeza de lograr el nivel de veracidad que sólo los matemáticos pueden garantizar: una ambición que será la bandera del empirismo lógico - como muestra la auto-denominación del movimiento.

La idea de un programa que establece con anticipación una serie no modificable de operaciones que de ser practicadas garantizan el conocimiento científico sobre cualquier argumento es, todavía hoy, uno de los significados que pre valecen en la expresión método científico. Pero - vistas también las dificultades encontradas por los empiristas lógicos a la hora de transferir aquellas ideas desde el plano de los enunciados de principio a sus aplicaciones prácticas en todos sus mínimos detalles - tal idea queda pospuesta antes de ocupar triunfalmente el escenario, como en los tiempos de Bacon y D escartes. Lo confirma, entre otras cosas, la creciente escasez de textos que se proponen especificar la mencionada serie no modificable de operaciones.

La más reciente - a mi entender- de dichas listas de operaciones que tiene, aún sin entrar en detalles, una cierta pretensión de complitud ha sido publicada por T heodorson y T heodorson en la voz «Scientific M ethod» en su Diccionario de Sociología (1970: 370). Dice así:

La aplicación del método científico a un problema comporta los pasos siguientes:

Primero: se define el problema.

Segundo: el problema se formula en los términos de un cuadro teórico determinado.

Tercero: se imaginan una o más hipotésis relativas al problema, utilizando los principios teóricos ya aceptados.

Cuarto: se determina el procedimiento a utilizar en la recolección de datos para controlar la hipótesis.

Q uinto: se recolectan los datos.

Sexto: se analizan los datos para averiguar si la hipótesis es verificada o rechazada. En fin, las conclusiones del estudio se relacionan con el corpus precedente de la teoría que se modifica, a su vez, para ajustarlo a los nuevos resultados.

Como el lector habrá observado, se trata de una descripción puntual del aś llamado «método hipotético-deductivo» elaborado a partir de la reflexión filosófica en torno a la física del siglo XVII en adelante (Losee, 1972, cap. 7-9). La convicción de que éste es el único método posible para la ciencia ha sido muy defendida incluso en las ciencias humanas (lo revelan, entre otros, Strasser, 1979 y Pérez Ransanz, 1995). H asta los años cincuenta no era nada raro encontrarse con declaraciones como la siguiente, del antropólogo N adel: «H ay un único método científico, a pesar de ser practicado con diversos niveles de rigor y coherencia, y a este respecto la física y la química han alcanzado los mejores 
resultados. Toda investigación está vinculada a este método, y no se puede concebir otro» (1949. D eclaraciones tan categóricas como ésta se pueden encontrar en las obras del psicólogo conductista Skinner (1953: 5), del sociólogo matemático Lundberg (1938: 191-192), del antropólogo M urdock (1949: 183) y - naturalmente - también en las obras de los epistemólogos neopositivistas ( $N$ eurath, 1931: 407; H empel, 1935, trad. ingl. 1948: 382; Feigl, 1963, trad. it. 1974: 211) o cercanos al neopositivismo (Kemeny, 1959; Rudner, 1966). En epistemología las tesis de la unidad del método científico ha sido afirmada también recientemente, de forma más cauta: «se puede razonablemente afirmar que las diversas disciplinas científicas tienen el mismo método en lo que se refiere a los procedimientos o al complejo de reglas que las integran» (Pera, 1978: 11; análogamente Bhaskar, 1979: 3).

Pero no todos están de acuerdo. D alton ha observado polémicamente: «se asume que una secuencia invariable: formulación de hipótesis/control/confirmación es compartida por todos los científicos, que todos concuerdan en que ésta es la única vía al conocimiento; en resumen, que existe un único método científico» (1964: 59). En cambio «cabe preguntarse - observa Becker- si los metodólogos, los guardianes institucionales de la metodología, afrontan realmente todo el espectro de las cuestiones metodológicas relevantes para la sociología, o si se limitan a un subconjunto no casual (como dirían ellos) de tales cuestiones». Luego concluye: «la metodología es demasiado importante como para ser dejada a los metodólogos!» (1970: 3). En el mismo sentido, el sociólogo y epistemólogo polaco M okrzycki dice: «L os fundamentos de este "método" se encuentran fuera de la sociología, carentes de contacto con el pensamiento sociológico. La "metodología de las ciencias sociales" se ha vuelto una correa de transmisión que distribuye el "método científico" a los sociólogos, es decir, las ideas de aquellos autores que pasan por ser expertos en el tema» (1983: 72). «M uchos de nosotros - agrega D alton - aceptamos el "método científico" porque estamos convencidos de que se ha desarrollado en las ciencias naturales [...] Pero en las ciencias naturales no se profesa deferencia a este modelo como hacemos nosotros» (1964: 59).

La vivacidad de estas reacciones se explica por su relación con un período, no lejano, en el cual la orientación cientista dominaba en las ciencias humanas: se afirmaba - como se ha visto en los párrafos citados- no sólo que la ciencia tiene un solo método, sino que se trata del método santificado por los éxitos de las ciencias físicas.

H a habido, y hay, posiciones más prudentes: «el método de una ciencia es la base lógica común sobre la cual se funda la aceptación o el rechazo de hipótesis y teorías» (Rudner, 1966, trad. esp. 1980). Esta posición minimalista - compartida por ejemplo por D ewey (1938) y por Popper (1944-45) - , según la cual el método es sólo uno porque se reduce al uso de la lógica en la argumentación, ha sido cuestionada por quien leía entre líneas «el supuesto de que el único método lógico es aquél utilizado en las ciencias naturales, y en la física en particular» (Schutz, 1954: 272). M ás severamente, también se han rechazado las tesis que reducen el núcleo unitario del método a la base lógica 
común, calificándola como «insignificantes generalizaciones que pretenden cubrir casi toda la actividad científica y en realidad cubren casi toda la actividad intelectual» (N ickles, 1986: 115).

Pero independientemente de las lecturas entre líneas (por lo demás plenamente fundadas, por lo menos en lo que respecta a Rudner, Popper y muchos otros), la propia idea de la unidad del método ha sido criticada. «La idea misma de "un solo método" acrecienta la confianza en los propios resultados y reduce la predisposición a preguntarse si los procedimientos consolidados tienen sentido en el caso particular» (K riz, 1988: 184). Para el epistemólogo francés H aroun Jamous, en la raíz de esta orientación se encuentra la «repulsión por la incerteza [...] la perenne necesidad de riendas estables y definitivas que puedan evitarnos el recurrir a aquella aportación individual e incierta que, justamente por ser difícilmente formalizable, es tal vez indispensable en toda obra creativa» (1968: 27).

Una segunda línea crítica, vinculada a la precedente, señala el peligro de que una determinada serie de procedimientos, identificada con «el método científico» se reifique convirtiéndose en un fin en sí misma. La situación «difundida en psicología [es] de exaltación y quasi-fetichismo del método eincluso del instrumento técnico que de medio se convierte en el fin de muchas investigaciones» (Parisi y Castelfranchi, 1978: 79).

«Como en todos los rituales, la atención pasa del contenido a la forma y la virtud consiste en la ejecución correcta de una secuencia fija de actos) (Kaplan, 1964: 146). «Si el criterio es el respeto a un sistema de reglas - observa maliciosamente Lecuyer - , incluso una guía telefónica es una buena investigación científica» (1968: 124).

Personalmente no llevaría la crítica tan lejos como el filósofo convencionalista Leroy cuando habla de «superstición» (1899: 377) o como el sociólogo Weigert cuando hace notar que «la característica de la magia es la atribución de eficacia a rituales minuciosamente observados sin indagar el nexo causal entre rito y efecto» (1970: 116).

Sin embargo, la naturaleza tendencialmente ritualista de la adhesión de muchos científicos sociales al «método científico» ha sido resaltada otras veces. Frecuentemente sucede que esa secuencia rigurosa de actuaciones aparece invariablemente en el momento de presentar los informes de investigación (M erton 1949: 506; Philips, 1966, lo que recuerda las punzantes observaciones de Fleck sobre el proceso de racionalización ex post que sufren los procedimientos experimentales (1935, § 4.2). Kaplan ironiza sobre «los prólogos metodológicos, antepuestos como invocaciones rituales a las deidades tutelares del método científico» (1964: 20).

Aún compartiendo muchas de estas críticas a la tesis de la natural eza única y fija del método científico, no se puede subscribir la boutade de los estadísticos Wallis y Roberts, para quienes «no hay nada que pueda llamarse "método científico". Es decir, no existen procedimientos que indiquen al científico cómo empezar, cómo proseguir, qué conclusiones alcanzar» (1956: 5). Aunque raramente explicitada en todo detalle, y objeto de una reverencia en gran medida 
ritual, hay en la conciencia colectiva de las investigaciones en ciencias humanas una cierta idea de que el método científico existe: se la encuentra, por ejemplo, en el recurso casi universal a palabras clave como hipótesis, verificación, experimental. Sin embargo, aun cuando la reverencia no es ritual, siguiendo esa imagen de método se llega frecuentemente a considerar objetos y problemas cognitivos sólo como si estuviesen reflejados en un prisma, a tomar esas imágenes deformadas por la realidad y a perder la ocasión de conocer esa realidad más de cerca, penetrándola con mayor profundidad. Tal vez debido a este tipo de motivos se ha afirmado que «el enorme florecimiento de escritos de filosofía de la ciencia ocurrido en el siglo xx, considerado en su conjunto, ha sofocado más que incentivado el desarrollo metodológico» (N ickles, 1986: 94-95).

«La calidad de un científico - recuerda Toulmin - se demuestra menos por su fidelidad a un método universal que por su sensibilidad a las exigencias específicas de un problema» (1972, vol. I: 150). En sus comentarios a la compilación de ensayos clásicos de investigación social, M adge sostiene: «si es justo pedir a un investigador que indique de la manera más precisa lo que pretende hacer antes de iniciar el trabajo, de ello no se deriva necesariamente que quienes hacen exactamente lo que habían establecido sean los mejores investigadores [...] Las virtudes del buen investigador son la flexibilidad, la prontitud para comprender una nueva situación y la capacidad para aprovechar las ocasiones imprevistas» (1962). Por sí misma, la fidelidad a los procedimientos codificados no ofrece ninguna garantía: «dado un procedimiento adecuado, es posible reconocer investigaciones consideradas pseudo-cientifícas que lo satisfacen» (se trata de la «paradoja del procedimiento» resaltada por Pera, 1991: 27). «Sea un buen artesano, que elige cada vez el procedimiento a seguir» recomienda M ills; y agrega una invitación: «pedimos a quien haya producido trabajos de calidad que nos cuente en detalle como ha operado: sólo en estas conversaciones con investigadores expertos los jóvenes podrán adquirir una sensibilidad metodológica adecuada» (1959: 224-228).

Son muchos los que dicen que el método es sobre todo una elección. «La cuestión metodológica propiamente dicha es la elección de la técnica en función de la naturaleza del tratamiento que cada técnica impone a su objeto» (Bourdieu y otros, 1968: 59). «C ada investigación es un largo sendero con muchas bifurcaciones donde se debe tomar una decisión [...] N inguna regla, ningún algoritmo puede decir cual es la decisión justa [...] El investigador cuanto más conciba el método como una sucesión rígida de pasos, más decisiones tomará sin reflexionar y sin darse cuenta» (Kriz, 1988: 81-131). Además, tener que escoger no significa necesariamente tener que afrontar cada problema con una única técnica: es más sabio no confiar en una sola técnica «para observar nuevos aspectos del fenómeno que interesa y tener en cuenta diversos tipos de datos relativos a un mismo fenómeno», de manera que se pueda «estar seguro de estudiar algo real», ya que de lo contrario, [el fenómeno] podría ser un artefacto de la técnica usada» (Parisi e Castelfranchi, 1978: 71). Incluso un metodólogo de orientación conductista como Van M eter no tiene dificultad alguna en admitir que «si se elige una sola técnica, los resultados no pueden ser con- 
siderados independientes de la técnica elegida, y por lo tanto no son estables: pueden de hecho ser contradecidos por resultados obtenidos con otras técnicas» (1994: 23).

Si la investigación debe ser «un proceso cognitivo más bien que un simple proceso de validación de ideas ya formuladas» (Bailyn, 1977: 101), es necesario «permanecer abiertos a nuevas informaciones e ideas, resistiendo a las inevitables presiones hacía interpretaciones prematuras» (Blaikie y Stacy, 1982: 32). 0 al menos «saber mantener la tensión entre la necesaria función de reducción de la complejidad fenoménica y la apertura a dimensiones que permitan aumentar la complejidad de los esquemas conceptuales» (C respi, 1985: 343).

El método es, por lo tanto, algo mucho más complejo que una simple sucesión de pasos unidimensionales (ver, por ejemplo, Cipolla, 1988). No basta, como admitía incluso Comte, «haber leído los preceptos de Bacon y el D iscours de D escartes» (1830, vol. I, ed. 1926: 71). «Sin duda - observa Polanyi- el científico procede de manera metódica. Pero su método es como las máximas de un arte que aplica de manera original a sus problemas (1958: 311). Su obra es «muy similar a una creación artística [...] pero no es un arte como la escultura y la pintura, en las cuales uno es libre de obtener cualquier cosa del material en bruto. Es un arte como la arquitectura, en el cual se puede mostrar creatividad trabajando con materiales en bruto, caracterizados por propiedades ingenieriles limitadas, y bajo financiaciones con presupuestos vinculados a objetivos precisos» (D avis, 1974: 267-268). La gestión de recursos sometida a objetivos es un aspecto característico del concepto de método, y volveremos sobre esto en el apartado 4.1.

Se puede concluir que la visión cartesiana-baconiana del método, que hasta hace algunos decenios no se ponía en tela de juicio, hoy en día genera perplejidad. No parece haber escandalizado a nadie M arcello Pera, cuando ha descrito la «paradoja del método»: «la ciencia se caracteriza por su método, pero una caracterización precisa del método destruye la ciencia» (1991: 39).

\section{M étodo, métodos, metodología}

El uso de «método/métodos» en el sentido de «técnica/técnicas» ha sido y es endémico en las disciplinas más orientada a la investigación empiríca, que están más frecuentemente en contacto con instrumentos operativos. N o obstante, se encuentran también muchos casos en obras de sociólogos téoricos. ${ }^{2}$

$\mathrm{H}$ ay autores que manifiestan un cierto malestar, y por ello hablan de «una técnica y método de análisis» (C apecchi, 1967: CLX) o de technical methods (C ollins, 1975: 414). Kaplan distingue los métodos de las técnicas por cuanto las primeras sirven de modo bastante general para todas las ciencias o en la

2. Véase, por ej., Sorokin (1947: 22); Znaniecki (1950: 220); H omans (1950: 40, 371-372; Riesman y otros (1950: 180); Alberoni (1967: 11); Goldthorpe y otros (1968: 8); Collins (1975: 8) y Sztompka (1979: 72). 
mayoría de ellas (1964: 23). Esta acepción se encuentra, en efecto, en el uso de una cierta estratificación jerárquica en el sentido indicado por Kaplan (Ios métodos son más generales y/o más nobles que las técnicas), pero ningún otro autor - por lo que sabemos - la ha codificado, y menos aún definido de al gún modo.

Se ha hablado de una expansión semántica indebida del termino método. Pero un abuso todavía más grave, como veremos en breve, lo padece el termino metodología. En docenas de términos científicos - muchos de los cuales han pasado al lenguaje ordinario- el sufijo logía es sinónimo de «discurso sobre», «estudio de». Por lo tanto, metodología debería ser el «discurso, estudio, reflexion sobre el método» - en una o varias de las acepciones que hemos visto.

En efecto, éste es el sentido aplicado por los autores que no han olvidado las raíces griegas de la terminología científica y metacientífica: «la metodología se encarga del análisis de los procedimientos científicos y de los instrumentos de investigación» (N ovak, 1976: XV); «metodología es la evaluación crítica de las actividades de investigación en relación con los estándares científicos» (Smelser, 1976: 3); «la metodología puede definirse como el conocimiento que resulta de una reflexión sobre los aspectos empíricos de la investigación» (Stoetzek, 1965). ${ }^{3}$

En las definiciones presentadas y en aquéllas análogas, el elemento método (es decir, el objeto estudiado por la metodología) es entendido de manera más bien amplia como un terreno vagamente definido entre la espistemología y las técnicas (ver apartado 5).

Sólo Lazarsfeld restringe la acepción del elemento método a algo similar a lo aquí presentado, en la parte final del apartado 2: «la metodología codifica las prácticas de la investigación para evidenciar aquello que merece ser tenido presente en ocasiones futuras.» (Lazarsfeld y R osenberg, 1955: 3); «la metodología examina las investigaciones para explicitar los procedimientos que fueron usados, los supuestos subyacentes, y los procedimientos explicativos ofrecidos». (Lazarsfeld y otros, 1972: XI). «Esta codificación de procedimientos pone en evidencia los peligros, indica las posibilidades dejadas de lado y sugiere eventuales mejoras. Además, hace posibles las generalizaciónes del conocimiento metodológico, transmitiendo las contribuciones específicas de un investigador al patrimonio de la comunidad científica» (Barton y Lazarsfeld, 1967).

C on una bella imagen, Lazarsfeld sintetiza su punto de vista: «a poesía es emoción a la cual se vuelve con ánimo tranquilo. Considero la metodología un volver al trabajo creativo con el mismo estado de ánimo» (1959). Aún no habiendo nunca definido directamente el término método, Lazarsfeld lo muestra, en estos pasajes, como una actividad creativa, a la par de M ills, Polanyi, 
D avisy otros - es más, muestra que su concepción de método es tan clara y sólida que resiste incluso la ampliación semántica que el término método sufre casi inevitablemente cuando se vuelve parte del término metodología.

D efiniendo metodología muchos sienten la necesidad de precisar que no se debe confundir con el método mismo, y menos aún con las técnicas. ${ }^{4}$ Esta precisión parecería superflua, porque confundir «X» con «el estudio de $X$ » constituye un error manifiesto. Sin embargo, no es en absoluto superflua: en la investigación social norteamericana - cuyos practicantes tienen generalmente una consciencia muy tenue de las raíces griegas del lenguaje científico - se ha difundido desde hace tiempo (y ha sido implacablemente exportada a otros continentes) la costumbre de llamar methodol ogy a la técnica. Es éste el abuso terminológico que se señalaba más arriba; del que son responsables incluso autores de notable sofisticación intelectual, como G altung (1967, por ej., p. 376). Se comprenden las protestas del interaccionista Blumer hacia esta «asombrosa inclinación a identificar la metodología con una limitada porción de su objeto de estudio» (1969: 22; también véase Sartori, 1984: 9).

A propósito de la metodología en sentido estricto, se debate si debe de ser una disciplina prescriptiva o descriptiva. A favor de la segunda alternativa se ha pronunciado con fuerza D ewey (1938); otros han resal tado que el estudio empírico de como trabajan los científicos es una tarea de la historiografía y de la sociología de la ciencia; la metodología debe ser prescriptiva (H olzner, 1964; N ickles, 1986: 107); debe «fijar y justificar un sistema de reglas tal que asegure la corrección de acciones y decisiones determinadas» (Pera, 1978: 7). «La metodología - escribe Bruschi- implica una concepción lógico-racional de la ciencia [...] El aspecto normativo que en la filosofía de la ciencia puede ser latente, aquí es manifiesto y directo [...] el metodólogo declara aquello que el investigador debe hacer para obtener ciencia» (1991: 38-9).

Se puede aceptar la idea de que la metodología sea, en última instancia, también una disciplina normativa, en cuanto da indicaciones acerca de lo que es oportuno hacer en cada situación específica. Pero tales indicaciones no deben hacerse en base a un modelo abstracto y general de ciencia, sino a la luz de lo que la historiografía y la sociología de la ciencia nos indican. Por ejemplo, mostrando que una cierta técnica, perfectamene adecuada en abstracto, es en realidad muchas veces portadora de graves sesgos; por ejemplo, avisando de que una insuficiente consideración de los procesos psicosocioantropológicos hace que su aplicación tiienda a mover las relaciones entre los sujetos observados y/o entre los observadores.

La contraposición descripción/prescripción encuentra un paralelismo en la distinción entre los dos significados que el término metodología tiene en el lenguaje corriente en las universidades y en la investigación social - significados que corresponden a los dos roles del metodólogo profesional- . Cuando estu- 
dia y enseña, el metodólogo debe de tener una orientación descriptiva, es decir, debe de estar abierto a aprender de las experiencias de otros investigadores evaluándolas sin prenociones, y estar dispuesto a referir de manera sistemática y sintética lo que ha aprendido. Cuando utiliza sus propias competencias, capacidad y experiencia al servicio de una investigación, suya o de otros, el metodólogo no puede ser más que prescriptivo, en tanto que debe elegir los instrumentos a utilizar y el cómo utilizarlos. En este sentido, será mejor que en sus prescripciones tenga en cuenta todo aquello que ha aprendido en su otro rol.

\section{M étodo y técnica}

También el término técnica deriva del griego, en el que $\tau \varepsilon \chi \nu \eta$ designa una capacidad artística, no aquélla individual e irrepetibile del genio, sino aquélla más doméstica, transmisible de padre a hijo, del artesano (en efecto, el término tiene un probable parentesco con $\tau \varepsilon \kappa v o v$, hijo).

La bella definición de $G$ allino («complejo más o menos codificado de normas y formas de proceder reconocido por una colectividad, transmitido o transmisible por aprendizaje, elaborado con el fin de desarrollar una actividad manual e intelectual de carácter recurrente [...] Un procedimiento extemporáneo, que no se reitera ni sufre forma de codificación alguna, no es una técnica aún cuando resulte, una sola vez, excepcionalmente ingeniosa y eficaz» (1978: 712-3) muestra que muchos elementos del significado original griego se han mantenido por lo menos en uno de los significados centrales del término.

Gallino toma, en efecto, el significado antropológico, muy difundido también en el lenguaje ordinario. Pero junto a ese significado, se pueden distinguir al menos otros dos, que tienen en común una relación instrumental entre su referente y la ciencia, pero que se distinguen por la dirección de tal relación. En el segundo significado, la técnica utiliza el conocimiento adquirido por las ciencias sobre la realidad para modificar algunos aspectos de la misma. En el tercer significado, es la ciencia la que usa las técnicas para conocer mejor al gún aspecto de su objeto. En ambos conceptos, y en particular en el último - él que aquí nos interesa- están presentes aspectos de la acepción del concepto ilustrado por $\mathrm{G}$ allino.

En el ámbito de la medicina, la clínica se sirve del conocimiento adquirido por la fisiología y la patología - que son ciencias- para curar a los enfermos, y es por lo tanto una técnica en el segundo significado. U na situación análoga se da en la ingeniería civil, cuando utiliza los conocimientos de estática y dinámica para fabricar edificios, carreteras, puentes. Es cierto - como hace notar C ini- que «la tecnología proporciona a la investigación científica contribuciones originales» (1990: XIV; análogamente Lecuyer, 1987: 65); pero la distinción es analítica: si en el ámbito de la investigación tecnológica se adquieren nuevos conocimientos sobre la realidad que se desea modificar, en ese momento se está haciendo ciencia. Además, decir que la técnica (en el segundo significado) aplica los conocimientos científicos para intervenir sobre la 
realidad no significa disminuirla; somos todos conscientes del hecho de que «la invención de un instrumento muchas veces ha marcado una época» (Jevons, 1874: 272).

Las técnicas de las que yo hablo aquí son instrumentales para los fines cognoscitivos de las diversas ciencias. Son «os procedimientos específicos empleados en una ciencia, o por un género particular de investigaciones, dentro de una ciencia [...] son las formas de cumplimentar el trabajo de una ciencia que están consideradas, por razones más o menos convincentes, aceptables por una comunidad científica. El adiestramiento científico tiene que ver en gran medida con la adquisición del dominio de las técnicas» (Kaplan, 1964: 19). En gran medida, pero no del todo, como advierte Lazarsfeld (1959) - y muchos, incluido Kaplan, estarían de acuerdo.

C omo observa $\mathrm{M}$ cl ver, el término técnica señala un cierto procedimiento 0 el instrumento que lo ejecuta (1942: 283); se puede agregar que indica también el conjunto de competencias prácticas (know how) necesarias para utilizar correcta y eficazmente tal instrumento. Este triple designatum del término suele ser frecuente incluso en la vida cotidiana.

La relación entre método y técnica (en el significado analizado en este apartado) puede ser clarificado con una imagen. Cada investigación tiene un objetivo cognoscitivo, es decir, busca mejorar, profundizar, articular el conocimiento sobre un cierto tema. Podemosimaginar este objetivo como si se tratara de un claro en una selva: se confía en que puede ser al canzado, se espera alcanzarlo, pero no se sabe exactamente donde está; y menos aún si se tienen ideas claras sobre como llegar. Podemos también imaginar que el investigador (y/o quien toma las decisiones sobre como proceder) parte de un punto cualquiera en el confín de la selva. Pocas veces es plenamente libre; general mente cuenta con ayudas pero también con limitaciones. $D$ ispone de fondos públicos y privados, y casi siempre tiene colaboradores más o menos expertos en varias tareas. Puede recurrir, si le parece oportuno, a instancias especializadas en la recolección de información y/o en el análisis de datos. Entre sus recursos cuenta también con el bagaje de conocimientos derivados de anteriores experiencias de investigación, propias y ajenas. La limitación principal viene dada por el plazo fijado para la entrega de los resultados de la investigación. M uchas veces este plazo es impuesto por un comitente que no tiene idea alguna sobre la complejidad de la tarea. A veces la naturaleza del comitente, o más genéricamente el clima político-cultural, imponen límites a la libertad de profundizar en una otra dirección. 0 tras veces la naturaleza del problema investigado pone límites éticos a las técnicas que se podrían usar.

La selva a atravesar es recorrida a través de espacios más o menos amplios, en varias direcciones, por senderos ya trazados, más o menos transitados: son las técnicas que otros investigadores han ideado, modificado, desarrollado. $\mathrm{N}$ aturalmente, es mucho más cómodo recorrer senderos ya trazados; pero no se sabe si nos conducirán al claro buscado o a al guna otra parte.

La tarea del investigador/metodólogo consiste en escoger el camino, teniendo en cuenta la naturaleza de los senderos presentes en el terreno, el tiempo y 
los recursos disponibles (en particular, el grado de adiestramiento/predisposición de sus colaboradores para manejar las diversas técnicas). En este sentido, su tarea se asemeja a la combinación de factores productivos que se realizan contando con restricciones y limitaciones, disponiendo de recursos escasos y de economías externas (Schumpeter, 1942). También el emprendedor toma decisiones a menudo: en cada momento debe elegir si confiar una cierta tarea a colaboradores externos o proceder por sí mismo pero siguiendo un sendero ya bien transitado, u otro sendero apenas trazado, o bien combinar varios senderos; decidirá también si recorrer fielmente estos senderos o intentar pequeñas variaciones del recorrido (modificaciones de técnicas existentes) o bien internarse en la selva, imaginando procedimientos nuevos, tal vez para confrontar los resultados con los de las técnicas ya existentes.

Lo esencial del concepto de método está en lo siguiente: en la elección de las técnicas a aplicar, en la capacidad de modificar técnicas existentes, adaptándolas a los propios problemas, y en imaginar técnicas nuevas. Una vez que un procedimiento nuevo, o una modificación de un procedimiento existente, han sido ideados y son codificados y difundidos, crsitalizan y se convierten en una técnica a disposición de la comunidad de investigadores: no es ya una capacidad privada, sino un objeto público. Sucede como con la parole de Saussure: una vez comunicada, ya es langue.

$\mathrm{N}$ aturalmente, los resultados de una investigación dependen del conjunto de elecciones que el investigador/metodólogo ha realizado a lo largo de todo el recorrido. Por este motivo, en el informe de investigación se debería dedicar amplio espacio para detallar y comentar las elecciones hechas, evaluando en lo posible sus impactos sobre los resultados. Lamentablemente, en cambio, es muy raro que este espacio exista (Schussler [1982] es una de las elogiables excepciones).

Como determinantes de esta situación - que demuestra una conciencia científica todavía embrionaria- influyen varios factores:

- El objetivismo del investigador, que cree debilitar el valor de sus resultados si admite cuánto dependen de las elecciones efectuadas (o que atribuye, con gran conocimiento de causa, tales convicciones a su público).

- El hecho de que buena parte del público se comporta como si leyese una novela policíaca: quiere saber quien es el asesino y no se interesa por los detalles del proceso investigador (C ohen y N agel, 1934: 399-400).

- Finalmente, el hecho de que los responsables de editoriales y revistas comparten en gran medida el objetivismo de autores y público y el hambre de hechos del segundo, cuestiones que deben tenerse en cuenta aunque no se compartan.

Sucede entonces que incluso autores muy escrupulosos reducen el informe metodológico a un apéndice, imaginando que «aquél que quiera saber más acerca de lo que el investigador ha hecho efectivamente y del modo en que lo ha hecho [...] dispondrá de tanta paciencia como de curiosidad»(Calvi, 1980: 21). 
El hecho de que las técnicas no posean la naturaleza intangible del método, sino que sean objetos tangibles y disponibles, no implica una menor nobleza o relevancia para el trabajo científico. La orientación de los grand theorists (como los Ilamaba irónicamente M ills, 1959) que desprecian las técnicas es precientífica, dado que sólo a través del empleo de técnicas se puede controlar empíricamente de algún modo una teoría - e incluso una gran teoría- . D e más está decir que sólo a través de un uso competente de las técnicas ese control se puede hacer de manera fiable.

Simétricamente, cae en una postura equivocada aquél que fetichiza una o más técnicas, es decir, aquél que desarrolla una gran pericia en su aplicación, pero las considera sólo como objetos ready made, carentes de historia y de profundidad: ignora y quiere ignorar el hecho que cada técnica ha sido ideada y desa rrollada en el marco de ciertos supuestos ontognoseoepistemológicos, ${ }^{5}$ que legitiman el recurso a determinadas manipulaciones de la información o a ciertas interpretaciones de los resultados.

D esde hace al gún tiempo, esta tendencia a la especialización rígida ha sido desvelada y denunciada en los EEUU (por ej., por Hill, 1970: 14): Ios metodólogos son en realidad especialistas en una o dos técnicas, y no están en condiciones de afrontar problemas más generales de la investigación. Se puede pensar que la superespecialización se desarrolla en paralelo a la tendencia al consumo de masas, porque presenta dos características estrechamente parecidas: la propensión a orientarse hacia el producto nuevo abandonando el antiguo cualquiera que fuera su valor, y la propensión a orientarse hacia productos todavía más artificiales y efectistas (que en nuestro caso quiere decir sobre todo computer intensive). Basta pensar en la rápida sucesión de modas que han traido a la escena del análisis de datos primero la regresión múltiple, luego el análisis factorial, luego el path analysis, luego los modelos loglineares, luego el análisis de las correspondencias, y así sucesivamente. Lo observaba de manera incisiva Labovitz: «Q ue en análisis de datos de las ciencias sociales imperen las modas es más que evidente. ¿Q uién sigue usando el escalograma de G utman? Esta técnica demodée ha sido substituida por otras [...] Se usa la técnica en boga, sea apropiada o no. La gente se pregunta: "quiero emplear el análisis factorial: ¿cual es el problema?" 0 bien te dice: "quiero utilizar el último grito de la moda, el path analysis: ¿Tienes al gunos datos?".» (1968: 221).

Se ha puesto como ejemplo el sector de análisis de datos porque ha sido el teatro de este reciente desarrollo. Ricolfi lo ha juzgado, un poco severamente, como un «progreso aparente» (1932: 338), y D avis piensa que «las técnicas estadísticas avanzadas no nos dicen mucho más que el análisis tabular de los tiempos de Lazarsfeld» (1987: 179). Pero, más allá del juicio sobre su utilidad, la opinión general es que las técnicas de elaboración matemáti-

5. Sobre como es la realidad, como podemos conocerla y cuales son los objetos y los límites de la ciencia. 
co-estadística se encuentran actualmente mucho más desarrollados que las técnicas de recolección de informaciones o de presentación gráfica de los resultados. ${ }^{6}$

Los textos de metodología, ironiza Troy D uster, siguen afirmando que todas las técnicas tienen el mismo valor... D e hecho, el programa de los cursos y el tipo de artículos aceptados para su publicación pronto dan el mensaje a estudiantes y doctorandos: ¡las técnicas están estratificadas!» (1981: 112).

$\mathrm{H}$ ace al gunos años $\mathrm{C}$ apecchi lamentaba «el desequilibrio entre el desarroIlo de estos métodos matemáticos y sus efectivas aplicaciones», sosteniendo que «el conocimiento de la matemática y de la estadística proporciona prestigio. Lo importante es que el investigador llegue a demostrar [...] que sabe dominar estos métodos» (1972: 39). También D avis dice que «sirven más que nada como medallas para exhibir» (1987: 179).

$\mathrm{N}$ aturalmente, podemos preguntarnos acerca del motivo de este mayor desarrollo, y/o mayor prestigio de las técnicas de análisis de datos - tal como ha afirmado tranquilamente $V a n$ M eter, que fue durante muchos años presidente del comité de metodología de la ISA, en una reseña dedicada por la revista de la U N ESC 0 a los diversos sectores de la sociología: «EI término método significa habitualmente 'método de análisis de datos'a menos que se especifique otra cosa» (1994: 19).

Se decía más arriba que la fascinación por las novedades es un motivo implícito en la tendencia a la especialización; sin embargo, ese mismo motivo no es válido por lo que respecta a las técnicas de análisis en su conjunto: en los departamentos de ciencias sociales norteamericanos y noreuropeos las técnicas estadísticas no son novedad en los currículos desde hace al menos treinta o cuarenta años. Sin duda, vale en cambio el otro motivo, es decir la propensión a procedimientos computer-intensive más bien que field-intensive (más similares a un juego electrónico que a un paseo con amigos por el bosque). Este factor actúa ya sea por fuerza propia, ya sea por vía de imitación de las ciencias físico-naturales, en las que las simulaciones informatizadas tienen un papel cada vez más importante: motivo por el cual, dicho factor parece destinado a ejercer una influencia duradera.

No se puede dejar de lado, por último, una explicación de corte kuhniano: buena parte de los jovenes emergentes en las ciencias humanas en los años sesenta y setenta han percibido la preparación estadística como el canal privilegiado para constituir rápidamente un patrimonio de expertise que sus mayores no poseían, lo que les permitía hacerse imprescindibles, y demandar a mediano plazo una sucesión anticipada (Collins, 1975: 54). 


\section{M etodología (y método) entre gnoseología y técnica}

Existe un consenso general sobre el hecho que la metodología ocupa «la porción central de un continuum de análisis crítico [...] [entre] el análisis de los postulados epistemológicos que hacen posible el conocimiento social y [...] la elaboración de las técnicas de investigación»(Gallino, 1978: 465). 0 cuparse de metodología es encontrarse en una continua tensión dialectica entre los polos de este continuum, porque «si la metodología abandona su lado epistemológico se reduce a una tecnología o una práctica que ya no controla intelectualmente. Si abandona el lado técnico, se transforma en una pura reflexión filosófica sobre las ciencias sociales, incapaz de incidir sobre las actividades de investigación» (Bruschi, 1991: 41).

Q ueda por añadir que en uno de los polos no está tan sólo la epistemología (reflexión sobre los fines, condiciones y límites del conocimiento científico), sino también la gnoseología (reflexión sobre los fines, condiciones y límites del conocimiento tout-court). La gnoseología ha sido y es frecuentemente olvidada en enunciados como los aquí mencionados. Las razones pueden ser dos, una lingüística y otra substancial. El término homónimo (sería gnoseology) no existe en inglés, y por lo tanto nunca aparece en la imponente literatura anglo-norteamericana sobre el tema. En inglés, de hecho, se usa epistemology para designar la filosofía del conocimiento («gnoseología») y predominantemente philosophy of science por lo que nosotros llamamos «epistemología».

El segundo motivo puede derivar del hecho que todos los intentos por proporcionar fundamentos de certeza absoluta al conocimiento científico (los más recientes el fenomenalismo de Schlick y el primer Carnap, y el fisicalismo de N eurath o el segundo Carnap) han fracasado frente a la constatación, inevitable en gnoseología, que no pueden haber nexos rígidos entre la esfera de los referentes (la realidad), la esfera del pensamiento y la esfera del lenguaje ( $M$ arradi, 1994). Puede suceder que esta situación, aceptada con escaso entusiasmo por muchos de los que se ocupan de ciencia, haya provocado un sentimiento de turbación frente a una disciplina que pone en evidencia los límites de las pretensiones cognoscitivas de las otras.

Si la metodología se sitúa donde se ha dicho, ¿dónde se sitúa el método? En el apartado 4 se ha planteado que el método consiste esencialmente en el arte de el egir las técnicas más apropiadas para enfrentar un problema cognoscitivo, eventualmente combinándolas, comparándolas, aportando modificaciones e incluso proponiendo alguna solución nueva. Lo que no surgió en las secciones precedentes y que quisiera subrayar antes de concluir estas reflexiones, es que el metodólogo no realiza estas elecciones sólo a la luz de sus competencias técnicas y de las experiencias de investigación propias y ajenas. Su propensión a utilizar una u otra técnica y su manera de interpretar las experiencias de investigación están condicionadas por sus opciones gnoseoepistemológicas: «Las soluciones técnicas presuponen soluciones metodológicas generales y éstas últimas, por otra parte, implican que se den respuestas adecuadas a ciertas 
cuestiones epistemológicas» (Ammassari, 1985: 178; véase también Gallino, 1978: 464).

\section{Bibliografía}

Alberon I, F. (1967). Introduzionea «Alberoni ealtri», L'attivista di partito. U n'indagine sui militanti di base nel Pci enella Dc, Bolonia, II M ulino.

Alexan Der, J. (1982). Theoretical Logic in Sociology, vol. I: Positivism, Presuppositions, and Current Controversies, Londres, Routledge \& Kegan Paul.

AM M ASSARI, P. (1985). « fondamentali problemi di metodologia della ricerca sociale», en Studi di sociologia, XXIII, 2-3, p. 176-93.

Arn Auld, A.; N ICOLE, P. Logique, ou art de penser, París 1662 [M adrid, Alfaguara, 1987].

BACHELARD, G. (1994). Le nouvel esprit scientifique, París, Alcan, 1934.

- (1938). La formation de l'esprit scientifique, París, Vrin. [M adrid, Siglo XXI, (20a ed).

BACON, F. (1620). N ovum Organum.

BAILYN, L. (1977). «Research as a Cognitive Process: Implications for D ata Analysis», en Q uality and Quantity, XI, 2, p. 97-117.

BARTO N , A. H .; LAZARSFELD, P. F. (1967). «Some Functions of Q ualitative Analysis in Social Research», en Frankfurter Beiträge zur Soziologie, I, p. 321-61 [Barcelona, Laia, 1979 ( $\left.2^{\mathrm{a}} \mathrm{ed}.\right)$ ].

BeCKER, H . S. (1970). Sociological Work: M ethod and Substance, Chicago, Aldine.

- (1972). «O bservation by Informants in Institutional Research», en Q uality and Quantity, VI, 1, p. 157-69.

Berka, K. (1983). M easurement. Its Concepts, T heories and Problems, D ordrecht, Reidel, 1983.

BhASKAR, R. (1979). The Possibility of Naturalism: A Philosophical Critique of the Contemporary H uman Sciences, Brighton, $\mathrm{H}$ arvester Press.

Blaikie, N. W. H.; Stacy, S. J. G. The D ialogical Generation of Typologies, M éjico, Xº Congreso Mundial de Sociología 1982.

BLALOCK, H. M. (1974). «ntroduction», en H. M. BLALOCK (ed.), M easurement in the Social Sciences. Theories and Strategies, Chicago, Aldine, p. 1-7.

Blum ER, H . (1969). Symbolic Interactionism: Perspectiveand M ethod, Englewood Cliffs, Prentice H all [Barcelona, H ora D.L., 1981].

Bo DIN, J. (1566). M ethodus ad facilem historiarum cognitionem, París.

BOLLEN, K. A.; J ACKM AN, R. W. (1990). «Regression Diagnostics: An Expository Treatment of $O$ utliers and Influential C ases", en J. Fox e J. S. Lo N G (eds.), M odern M ethods of D ata Analysis, Londres, Sage, p. 257-291.

Bourdieu, P.; Cham Bored on, J.-C.; PAsSeron , J.-C . (1968). Le métier de soci ologue. Problèmes épi stémologiques, París, M outon [M adrid, Siglo XXI, 1989 (12a ed.)].

BruschI, A. (1991). «Logica e metodologia», en Sociologia e ricerca sociale, XII, 35, p. 30-55.

Calvi, G . (1980). La classe fortezza. Scelte degli elettori eresponsabilità della classe politica in Italia, M ilán, Angeli.

CAPECCHI, V. (1967). «M etodologia e ricerca nell'opera di Paul F. Lazarsfeld», introduzionea P. F. LAZARSFELD, M etodologia e ricerca sociologica, Bolonia, II M ulino. p. VII-CLXXXII. 
CAPECCHI, V. (1972). «Struttura e tecniche della ricerca», en P. RossI (ed.), Ricerca sociologica e ruolo del sociologo, Bolonia, II Mulino, p. 23-120.

Cinı, M . (1990). Trentatré variazioni su un tema. Soggetti dentro e fuori la scienza, Roma, Editori Riuniti.

CipolLA, C. (1988). Teoria della metodologia sociologica. Una metodologia integrata per la ricerca sociale, M ilán, Angeli.

Cohen, M. R.; N agel, E. (1934). An Introduction to Logic and Scientific M ethod, N uevaYork, H arcourt [Buenos Aires, Amorrortu, 1968].

Collin S, R. (1975). Conflict Sociology: Toward an Explanatory Science, N ueva York, Academic Press.

Comte, A. (1926). Cours de philosophie positive, París, Rouen, 1830-42.

C O OLEY, C. H. (1909). Social O rganization, N ueva York, Scribner.

CRESPI, F. (1985). Le vie della sociologia. Problemi, teorie, metodi, Bolonia, II M ulino. D ALton, M . E. (1964). «Preconceptions and M ethods in 'M en W ho M anage'», en P.

E. H AM M ON D (ed.), Sociologists at Work, N ova York, Basic Books, p. 58-110.

D AVIS, J. A. (1964). «G reat Books and Small Groups: An Informal H istory of a N ational Survey», en P. E. H Am M ON D (ed.), Sociologi sts at Work, N ueva York, Basic Books, p. 244-269.

- (1987). «T he Future Study of Public O pinion», en Public O pinion Q uarterly, LI, 4, 1987, p. 178-179.

D ESCARTES, R. (1628). Regulae ad directionem ingenii.

- (1637). D iscours de la méthode.

DEWEY, J. (1938). Logic, the Theory of Inquiry, N ueva York, H olt.

DIRENZO, G. J. (1966). «Toward Explanation in the Behavioral Sciences», en G.J.

Direnzo (ed.), Concepts, Theories, and Explanation in the Behavioral Sciences, Nueva York, Random, p. 231-291.

DuncAN, O . D . (1984). N otes on Social M easurement. Historical and Critical, Nueva York, Russell Sage Foundation.

D uster, T. (1981). «ntermediate Steps Between M icro- and M acro-Integration: the C ase of Screening for Inherited D isorders», en K. D. KN ORR-C etin A y A. Cicourel (ed.) Advances in Social Theory and M ethodology, Londres, Routledge \& Kegan Paul, p. 109-135.

FEIGL, H . (1974). «Physicalism, U nity of Science and the Foundations of Psychology», en P. A. SchILPP (ed.), The Philosophy of Rudolf Carnap, vol. I, La Salle, O pen Court, 1963.

FLECK, L. (1935). Entstehung und Entwicklung einer wissenschaftlichen Tatsache. Einführung in die Lehre vom D enkstil und D enkkollektiv, Basel, Benno Schwabe.

G albraith , J. K. (1973). The Affluent Society, Boston, H oughton-M ifflin, 1958 [Barcelona, Ariel (3a ed.)].

Gallin O, L. (1978). Dizionario di Sociologia, Turín, UTET, (voces N eopositivismo e sociologia y Tecnica).

GaLtU N G, J. (1967). Theory and M ethods of Social Research, Londres, Allen \& Unwin [Buenos Aires, EU DEBA, 1973].

GIDDEN S, A. (1979). Central Problems in Social Theory. Action, Structure and Contradiction in Sociological Analysis, Londres, M acM illan.

GoLDTH ORPE, J. y otros (1968). The Affluent Worker: Political Attitudes and Behaviour, Cambridge University Press.

H EM PEL, C. G. (1935). «L'analyse logique de la psychologie», en Revue de Synthèse. 
H ILL, R. J. (1970). «O n the Relevance of M ethodology», en N . K. D EN ZIN (ed.), Sociological M ethods, Chicago, Aldine, p. 12-26.

H OBH OUSE, L. T. (1906). M oralsin Evolution. A Study in ComparativeEthics, Londres, Butterworths.

H OLT, R.; TURNER, J. E. (1970). «T he M ethodology of Comparative Research», en R. T. H OLT YJ. E. T URNER (ed.), The M ethodology of Comparative Research, N ueva York, p. 1-20.

H OLZN ER, B. (1964). «M ethodology», en J. Gould y W. L. Kolb (ed.), A D ictionary of the Social Sciences, Glencoe, Free Press, p. 425-426.

H om AN S, G . C. [1971]. The H uman Group, Nueva York, H arcourt, Brace, 1950 [Buenos Aires, EU DEBA].

Jam ous, H. (1968). «Technique, méthode, épistémologie», en Epistémologie Sociologique, 6, p. 21-38.

Jevons, W. S. (1874). The Principles of Science A Treatise on L ogic and Scientific M ethod, Londres, M acM illan.

KapLAN, A. (1964). The Conduct of Inquiry, San Francisco, Chandler.

Kem ENY, J.G . (1959). A Philosopher Looks at Science, Princeton University Press.

KRIZ, J. (1988). Facts and Artefacts in Social Science. An Epistemological and M ethodol ogi cal Analysis of Empirical Social Science Research Techniques, N ueva York, M acGraw-Hill, 1988.

LABOVITZ, S. (1968). «C riteria for Selecting a Significance Level: A N ote on the Sacredness of.05», en American Sociologist, III, 3, p. 220-222.

LaZARSFELD, P. F. (1959). «Problems in M ethodology», en R. K. M erton, L. Broo M y L. S. C ottrell (ed.), Sociology Today: Problemsand Prospects, N ueva York, Basic Books, p. 39-78.

Lazarsfeld, P. F.; Pasanella, A. K.; Rosen berg, M . (1972). «Preface», en Lazarsfeld, Pasanella y Rosen berg (ed.), Continuities in the Language of Social Research, N ueva York, Free Press.

LaZARSFeld, P. F.; Ro Sen Berg, M . (1955). «ntroduction» en P. F. LaZARSFeld y M . Rosen BERg (ed.), The Language of Social Research, N ueva York, Free Press, p. 1-12 [R. Bou d on-P. LAZARSFELD, M etodología de las Ciencias Sociales, Barcelona, Laia, 1985 (3a ed.)].

LECUYER, B.-P. (1968). «H istoire et sociologie de la recherche sociale empirique: problè mes de théorie et de méthode», en Epistémologie Sociologique, 6, p. 119-131.

LECUYER, B.-P. (1987). «Società post-industriale e professionalizzazione degli scienziati», en Sociologia e ricerca sociale, VIII, 24, p. 51-67.

LEROY, E. (1899). «Science et philosophie», en Revue de métaphysique et morale, VII, p. 364-381.

LOSEE, J. (1972). A H istorical Introduction to the Philosophy of Science, O xford U niversity Press [M adrid, Alianza, 1985 (4⿳亠丷a ed.)].

LUN D BERG, G. A. (1938). «T he C oncept of Law in the Social Sciences», en Philosophy of Science, V, 2, p. 189-203.

LUTYN SKI, J. (1988). «Un centro di ricerca sulle tecniche di raccolta dei dati», en A. M ARRADI (ed.), Costruireil dato, M ilán, Angeli, 1988, p. 173-187.

LYN D, R.; LYN D, H . (1929). M iddletown: A Study in Contemporary American Culture, N ueva York, $\mathrm{H}$ arcourt.

M ADGE, J. (1962). The O rigins of Scientific Sociology. N ueva York, Free Press.

M ARRADI, A. (1994). «R eferenti, pensiero e linguaggio: una questione rilevante per gli indicatori», en Sociologia e ricerca sociale, XV, 43, p. 137-207. 
M Clver, R. M . (1942). Social Causation, Boston, Ginn.

M CKen N ELL, A. C. (1973). «Surveying Attitude Structures: A Discussion of Principles and Procedures», en Q uality and Q uantity, VII , 2, p. 203-294.

M ERTON, R. K. (1980). Social Theory and Social Structure. Toward the Codification of

Theory and Research, Glencoe, Free Press, 1949 [M éxico, FCE, (2ª ed.)].

M ILLS, C. W. (1959). The Sociological Imagi nation, O xford U niversity Press [M éxico,

FCE, 1969 (3a ed.)-Barcelona, H erder, 1992].

M OKRZYCKI, E. (1983). Philosophy of Science and Sociology. From the M ethodological

D octrine to Research Practice, Londres, Routledge \& K egan Paul, 1983.

M URD OCK, G. P. (1949). Social Structure, N ueva York, M acM illan.

N ADEL, S. F. (1949). TheFoundations of Social Anthropology, Londres, Cohen \& West

[M éxico, FCE, 1974].

N EURATH, O . (1931-32). «Soziologie im Physikalismus», en Erkenntnis, II, p. 393431.

N ICKLES, T. (1986). «M etodologia, euristica e razionalità», en M . Pera y J. PITt (ed.), I modi del progresso: teorie e episodi della razionalità scientifica, M ilán, II Saggiatore, p. 87-116.

N owAK, S. (1976). Understanding and Prediction. Essays in the M ethodology of Social and

Behavioral Theories, Dordrecht, Reidel.

N owAK, S. (1979). «M ethodological D ilemmas of Contemporary Sociology», en J. J.

W IATR (ed.), Polish Essays in the M ethodology of the Social Sciences D ordrecht, Reidel, p. 157-171.

O PP, K. D . (1970). M ethodologie der Sozialwi ssenschaften, H amburg, Reineck.

PARISI, D .; CASTELFRANCHI, C. (1978). «Una definizione della psicologia cognitivis-

ta», en G. KAN IZSA y P. LEGRENZI (ed.), Psicologia della gestalt e psicologia cogniti-

vista Bolonia, II M ulino, p. 63-84.

PARK, R. E.; BuRgess, E. W. (1921). Introduction to the Science of Sociology, Chicago

University Press.

PARSO N S, T. (1937). The Structure of Social Action. A Study in Sociological Theory with

Special Reference to a G roup of Recent European Writers, N ueva York, M cG raw-H ill

[M adrid, Guadarrama, 1968].

Pera, M . (1978). Induzione e metodo scientifico, Pisa, Editrice Tecnico-Scientifica.

- (1991). Scienza e retorica, Bari, Laterza.

Pérez Ran SAnZ, A. R. (1995). «Racionalidad y desarrollo científico», en León Olive,

Racionalidad epistémica, M adrid, Trotta.

PHILLIPS, B. S. (1972). Social Research. Strategy and Tactics, Londres, M acM illan, 1966.

PoLANYI, M . (1958). Personal Knowledge. Towards Post-Critical Philosophy, Londres,

Routledge \& Kegan Paul.

POPPER, K. R. (1944). «T he Poverty of H istoricism», en Economica, XI, 42, p. 86-103;

43, 1944, p. 119-137; XII, 46, 1945, p. 69-89 [M adrid, Alianza, 1984 (3a ed.)].

Rıco LFI, L. (1982). «M odelli di analisi e software per le scienze sociali», en Rassegna ita-

liana di sociologia, XXIII, 3, p. 331-372.

Riesm AN, D .; G LAZER, N .; D EN NEY, R. (1950). The Lonely Crowd. A Study of the

Changing American Character, N ew H aven, Yale University Press [Barcelona, Paidós, 1981].

Rudner, R. S. (1968). Philosophy of Social Science, Englewood Cliffs, Prentice-H all, 1966 [M adrid, Alianza, 1980 ( 2 a ed.)].

SARto RI, G. (1984). «Foreword» en G. SARTo RI (ed.), Social Science Concepts. A

Systematic Analysis, Londres, Sage. 
Saussu Re, F. D e (1916). Cours delinguistique générale, París, Payot [M adrid, Alianza, 1983-Barcelona, Ed. 62, 1990].

SCh U M PETER, J. A. (1942). Capitalism, Socialism, and D emocracy, N ueva York, H arper \& Row [M adrid, Aguilar, 1971-Barcelona, Ed. 62, 1989].

SCH USSLER, K. (1982). M easuring Social Life Feelings, San Francisco, Jossey-Bass, 1982.

SchuTZ, A. (1954). «C oncept and Theory Formation in the Social Sciences», en Journal of Philosophy, LI, 9, p. 257-273.

Selvin, H . C . (1958). «D urkheim's "Suicide" and Problems of Empirical Research», en American Journal of Sociology, LXIII, p. 607-619.

SkIN N ER, B. F. (1953). Science and H uman Behavior, G lencoe, Free Press [Barcelona, Fontanella, 1981].

Sm elSer, N . J. (1976). Comparative M ethodsin the Social Sciences, Englewood C liffs, Prentice $\mathrm{H}$ all.

So m ERS, R. H . (1972). «Applications of an Expanded Survey Research M odel to Comparative Institutional Studies», en I. VALLIER (ed.) Comparative M ethods in Sociology, Berkeley, University of California Press, p. 357-420.

So Ro KIN, P. A. (1947). Society, Culture, and Personality: Their Structure and D ynamics. A System of General Sociology. N ueva York, H arper [M adrid, Aguilar, 1966 (3a ed.)].

Statera, G . (1968). La conoscenza sociologica. Problemi e metodo, Roma, Carucci. Stoetzel, J. (1965). «Préface» a R. Boud on y P. F. LazARSFeld (ed.), M éthodes de la sociologie París, M outon [Boudon-Lazarsfeld, 1985].

St rasSer, C. (1979). La razón científica en política y sociología. Buenos Aires, Amorrortu. SzTo m PKA, P. (1979). Soci ological D ilemmas. Toward a D ialectic Paradigm, N ueva York, Academic Press.

THEODORSON, G.A.; THEOdORSON, A. G . (1970). A M odern Dictionary of Sociology, Londres, M ethuen.

TOULM IN, S. E. (1972). H uman U nderstanding, vol. I: T he Evolution of Collective U nderstanding, Princeton U niversity Press [M adrid, Alianza, 1977].

Van M eter, K. (1994). «Sociological M ethodology», en International Social Science Journal XLVI, 139, p. 15-25.

VoN W RIGHT, G. H. (1971). Explanation and Understanding, Ithaca, Cornell University Press [M adrid, Alianza, 1980].

W allis, W. A.; RoberTs, H . V. (1956). Statistics. A N ew Approach, N ueva York, Free Press.

W eBer, M . (1922). Gesammelte Aufsätze zur W issenschaftslehre, T übingen, M ohr. W EIGERT, A. J. (1970). «The Immoral R hetoric of Scientific Sociology», en American Sociologist, V, p. 110-123.

ZN ANIECKI, F. (1950). «European and American Sociology After Two World Wars», en American Journal of Sociology, LVI, 3, p. 217-221. 